This item was submitted to Loughborough's Research Repository by the author.

Items in Figshare are protected by copyright, with all rights reserved, unless otherwise indicated.

\title{
Rapid analysis of anabolic steroid metabolites in urine by combining field asymmetric waveform ion mobility spectrometry with liquid chromatography and mass spectrometry
}

\section{PLEASE CITE THE PUBLISHED VERSION}

http://dx.doi.org/10.1021/acs.analchem.7b00940

\section{PUBLISHER}

(C) American Chemical Society (ACS)

\section{VERSION}

AM (Accepted Manuscript)

\section{PUBLISHER STATEMENT}

This work is made available according to the conditions of the Creative Commons Attribution-NonCommercialNoDerivatives 4.0 International (CC BY-NC-ND 4.0) licence. Full details of this licence are available at: https://creativecommons.org/licenses/by-nc-nd/4.0/

\section{LICENCE}

CC BY-NC-ND 4.0

\section{REPOSITORY RECORD}

Arthur, Kayleigh L., Matthew Turner, Alan D. Brailsford, Andrew T. Kicman, David A. Cowan, Jim Reynolds, and Colin Creaser. 2017. "Rapid Analysis of Anabolic Steroid Metabolites in Urine by Combining Field Asymmetric Waveform Ion Mobility Spectrometry with Liquid Chromatography and Mass Spectrometry". Loughborough University. https://hdl.handle.net/2134/26205. 


\section{LC-FAIMS-MS Steroids}

Rapid analysis of anabolic steroid metabolites in urine by combining field asymmetric waveform ion mobility spectrometry with liquid chromatography and mass spectrometry

Kayleigh L. Arthur ${ }^{1}$, Matthew A. Turner ${ }^{1}$, Alan D. Brailsford ${ }^{2}$, Andrew T. Kicman², David A. Cowan ${ }^{2}$, James C. Reynolds ${ }^{1 \star}$, Colin S. Creaser ${ }^{1 *}$

${ }^{1}$ Centre for Analytical Science, Department of Chemistry, Loughborough University, Loughborough, Leicestershire, LE11 3TU, UK

2 Drug Control Centre, King's College London, London, SE1 9NH, UK

* Joint corresponding authors:

Colin S. Creaser: c.s.creaser@lboro.ac.uk

James C. Reynolds: j.c.reynolds@lboro.ac.uk 


\begin{abstract}
The combination of field asymmetric waveform ion mobility spectrometry with liquid chromatography-mass spectrometry (LC-FAIMS-MS) has been developed for the analysis of glucuronide and sulfate metabolites of seven anabolic-androgenic steroids AAS in urine. Separation by FAIMS-MS was investigated in positive ion mode for selected cationic adducts $\left(\mathrm{H}^{+}, \mathrm{NH}_{4}{ }^{+}, \mathrm{Na}^{+}, \mathrm{K}^{+}, \mathrm{Cs}^{+}\right)$. LC-FAIMS-MS analysis of the doubly sodiated adducts $\left([\mathrm{M}+2 \mathrm{Na}-\mathrm{H}]^{+}\right)$of isobaric and co-eluting steroid metabolites allowed their rapid (8 min) qualitative and quantitative determination in spiked urine using hydrophilic interaction liquid chromatography prior to FAIMS-MS separation, with discrimination $>95 \%$ achieved between the steroids investigated. A quantitative evaluation of the LC-FAIMS-MS method was performed giving limits of detection in the range 1-6 $\mathrm{ng} \mathrm{mL} \mathrm{m}^{-1}$, limits of quantification in the range 3-20 $\mathrm{ng} \mathrm{mL}^{-1}$, with reproducibility (\%RSD $<10 \% ; n=6)$ and linearity $\left(R^{2}>0.99\right)$. The LC-FAIMS-MS method demonstrates increases in signal-to-noise ratios for the doubly sodiated steroid metabolites in unspiked urine $(>250 \%)$ by the reduction of isobaric interferences from the matrix. An alternative or additional tool for identification of the steroid metabolites is based on the observations of different patterns of sodium acetate clusters that are characteristic for each metabolite.
\end{abstract}

\title{
Keywords
}

Field asymmetric waveform ion mobility spectrometry, FAIMS, Differential mobility spectrometry, Liquid chromatography, Mass spectrometry, Anabolic-androgenic steroid metabolites, Urine 


\section{Introduction}

The determination of anabolic androgenic steroids (AAS), either as free drugs or their glucuronide or sulfate metabolites, has conventionally used mass spectrometric based techniques including gas chromatography-mass spectrometry (GC-MS) and liquid chromatography-mass spectrometry (LC-MS). ${ }^{1}$ GC-MS remains the method of choice for targeted quantitative analysis of endogenous AAS (EAAS) and the ratios of their naturally-occurring isomers. ${ }^{2}$ The detection of EAAS is complicated by their natural presence in the body, which means the simple presence of these steroids and/or their metabolites cannot be used to prove administration. Population derived thresholds of steroid concentrations for detecting EAAS administration are limited in their sensitivity due to the large inter-individual variations, differences in the relative concentrations of the steroids to each other, the influence of urinary dilution, and the effect of confounding factors. For example, a study of over 3000 individuals found that in males (with concentrations typically higher than females) the mean concentrations for androsterone and etiocholanolone were $2640 \mathrm{ng} \mathrm{mL}^{-1}$ (max $\left.20,700 \mathrm{ng} \mathrm{mL}^{-1}\right)$ and $1850 \mathrm{ng} \mathrm{mL}^{-1}\left(11,400 \mathrm{ng} \mathrm{mL}^{-1}\right)$ respectively. ${ }^{3}$ For testosterone and epitestosterone the concentrations were much lower, $37.4 \mathrm{ng} \mathrm{mL}^{-1}$ ( $\max 249 \mathrm{ng}$ $\mathrm{mL}^{-1}$ ) and $28.9 \mathrm{ng} \mathrm{mL}^{-1}$ ( $\left.\max 391 \mathrm{ng} \mathrm{mL}^{-1}\right)$.

GC-MS may be the current method of choice for EAAS analysis, however, shortcomings of GC-MS are the need for derivatisation of the steroids prior to analysis, ${ }^{4}$ and long analysis times to resolve isobaric species. LC-MS does not usually require a derivatization step and can be used to enhance the speed of analysis with shorter chromatographic run times compared to GC-MS. ${ }^{4}$ Further specificity and sensitivity can be achieved by combining both chromatographic methodologies with tandem mass spectrometry (MS/MS) in targeted analysis. ${ }^{5,6}$ However, limitations of this approach include the inability to separate isobaric interferences by MS with similar retention times, and the identification of isobaric steroids with similar fragmentation patterns using MS/MS.

Ion mobility spectrometry (IMS) is an orthogonal separation technique that has been shown to be a powerful tool for distinguishing isomers of many different biological classes, including metabolites, ${ }^{7,8}$ peptides, ${ }^{9}$ lipids, ${ }^{10}$ and carbohydrates. IMS has also been shown to reduce chemical noise due to isobaric interferences in biological matrices when combined with LC- MS (and MS/MS). ${ }^{7,12,13}$ Established IMS techniques include static field drift tube IMS (DTIMS) and travelling wave IMS (TWIMS) systems, in which ions are separated on the basis of their velocity through the device in the presence of a buffer gas, the velocity being related to ion size and shape (collision cross section).

Field asymmetric waveform ion mobility spectrometry (FAIMS), also known as differential ion mobility spectrometry (DMS), is a gas-phase ion separation technique which separates ions based upon compound differences in the mobility of an ion through an alternating RF waveform, known as the dispersion field (DF), of high and 
low electric field strengths. A small DC voltage, known as the compensation field (CF), is used to correct ion trajectory allowing selected ions to pass through the device. The CF can be scanned at fixed DF in order to produce a FAIMS spectrum.

FAIMS has a high degree of orthogonality to both MS and LC, and has been shown to separate steroid isomers and isobars, whilst also increasing signal-to-noise ratios $(\mathrm{S}: \mathrm{N})$ for targeted compounds by removing isobaric interferences from biological matrices, such as plasma and urine. ${ }^{14-16}$ However, some of these reported studies required derivatisation prior to IMS or FAIMS analysis to achieve separation of the steroid isomers of interest. ${ }^{12,16}$ Here, we describe an LC-FAIMS-MS method using a miniaturized FAIMS device for the determination in urine of seven steroid metabolites of interest to anti-doping. ${ }^{17}$ The steroids targeted (Figure 1 ) include isobaric steroid sulfates: testosterone sulfate (TS), epitestosterone sulfate (ETS), and dehydroepiandrosterone sulfate (DHEAS); and two pairs of isobaric steroid glucuronides: testosterone glucuronide (TG), epitestosterone glucuronide (ETG) and androsterone glucuronide (ADG), etiocholanolone glucuronide (ECG). These pairs of steroid glucuronides and trio of steroid sulfate metabolites are exact mass isomers which cannot be separated by even the highest resolution mass spectrometry. The data presented here includes a FAIMS-MS investigation into the separation of the steroid isomers in positive ion mode The LC-FAIMS-MS method for the determination of the steroid metabolites uses a HILIC separation with an analysis time of less than 10 minutes. 


\section{Experimental}

\section{Chemicals}

HPLC grade acetonitrile (ACN), HPLC grade water $\left(\mathrm{H}_{2} \mathrm{O}\right)$, ammonium acetate, and sodium acetate were all purchased from Fisher Scientific (Loughborough, UK). Acetic acid was purchased from Sigma Aldrich (Dorset, UK), and cesium chloride was obtained from Riedel-de Haën (Seelze, Germany). Testosterone glucuronide ( $\mathrm{TG}, 50 \mu \mathrm{g} \mathrm{mL}-1$ ), epitestosterone glucuronide (ETG, $100 \mu \mathrm{g} \mathrm{mL}^{-1}$ ), etiocholanolone glucuronide (ECG, $87 \mu \mathrm{g} \mathrm{mL}^{-1}$ ), androsterone glucuronide (ADG, $100 \mu \mathrm{g} \mathrm{mL}^{-1}$ ), testosterone sulfate (TS, $77 \mu \mathrm{g} \mathrm{mL}-1$ ), epitestosterone sulfate (ETS $76 \mu \mathrm{g} \mathrm{mL}^{-1}$ ) and dehydroepiandrosterone sulfate (DHEAS, solid) solutions in methanol, were supplied by the Drug Control Centre (King's College London, London, UK). Anonymized urine samples were also supplied by the Drug Control Centre (King's College London, London, UK) with the urinary supernatants then stored at $-20^{\circ} \mathrm{C}$ prior to analysis.

\section{Sample preparation}

Standard solutions of the steroid compounds for FAIMS-MS $\left(2 \mu \mathrm{g} \mathrm{mL}^{-1}\right)$ and LCFAIMS-MS analysis were prepared by dilution in the starting LC mobile phase 98:2 v/v ACN: $\mathrm{H}_{2} \mathrm{O}$ with $10 \mathrm{mM}$ ammonium acetate and $0.1 \%$ acetic acid, with the addition of sodium acetate (3.69 $\left.\mathrm{g} \mathrm{m} \mathrm{m}^{-1}\right)$ for FAIMS-MS analysis. Standard solutions for LCFAIMS-MS and LC-MS analysis were prepared without the addition of sodium acetate. For spiking urine aliquots, the steroid standards were diluted in $\mathrm{H}_{2} \mathrm{O}$ and spiked $\left(1 \mu \mathrm{g} \mathrm{mL}^{-1}\right)$ into a pooled urine sample prior to sample clean-up. Investigations into the steroid metabolite FAIMS separation in the starting mobile phase were performed with the addition of sodium acetate $\left(3.69 \mu \mathrm{g} \mathrm{m}^{-1}\right)$ and cesium chloride $(0.34 \mu \mathrm{g} \mathrm{mL}-1)$.

Aliquots of urine $(500 \mu \mathrm{L})$, stored at $-80^{\circ} \mathrm{C}$, were thawed for twenty minutes. The urine was diluted with $\mathrm{H}_{2} \mathrm{O}(500 \mu \mathrm{L})$ or, for spiked aliquots, a mixture of all seven steroids diluted in $\mathrm{H}_{2} \mathrm{O}$ was added $(1 \mu \mathrm{g} \mathrm{mL}-1,500 \mu \mathrm{L})$ and the diluted urine $(1 \mathrm{~mL})$ was centrifuged for $10 \mathrm{~min}$ at 10,000 $\mathrm{g}$. A solid phase extraction (SPE) sample clean-up was employed, using a C18 cartridge (60 mg, Phenomenex, Macclesfield, UK) conditioned with $\mathrm{ACN}(1 \mathrm{~mL})$ and equilibrated with $\mathrm{H}_{2} \mathrm{O}(1 \mathrm{~mL})$. The diluted urine (1 mL) was loaded onto the SPE cartridge. The cartridge was washed with 14:86 v/v $\mathrm{ACN}: \mathrm{H}_{2} \mathrm{O}(1 \mathrm{~mL})$ before elution using $\mathrm{ACN}(2 \mathrm{~mL})$. The eluent was evaporated to near dryness $(-10 \mu \mathrm{L})$ at $30^{\circ} \mathrm{C}$ using a Turbovap LV concentration workstation (Caliper Life Sciences, Hopkinton, MA, USA) and reconstituted in $50 \mu \mathrm{L}$ 98:2 v/v $\mathrm{ACN}: \mathrm{H}_{2} \mathrm{O}$ with $10 \mathrm{mM}$ ammonium acetate and $0.1 \%$ acetic acid. The reconstituted urine extract was transferred to a polypropylene micro-insert (Agilent Technologies, Santa Clara, USA) in an autosampler vial (2 mL) for LC-FAIMS-MS and LC-MS analysis. 


\section{Instrumentation}

LC-FAIMS-MS and LC-MS analyses of steroid metabolites were performed using an Agilent 1200 series HPLC coupled with an Agilent 6230 time-of-flight (TOF) mass spectrometer fitted with a JetStream electrospray ionisation (ESI) source operated in positive ion mode (Agilent Technologies, Santa Clara, USA). The miniaturized FAIMS device (ultraFAIMS, Owlstone Ltd.), with an electrode gap of $100 \mu \mathrm{m}$, a short trench length $(78.1 \mathrm{~mm})$ and ion path length $(700 \mu \mathrm{m})$, which has been described elsewhere, ${ }^{18,19}$ was located in front of the inlet capillary of the mass spectrometer behind a modified spray shield in the ESI source. LC-MS analyses were performed with the FAIMS device in place and no electric field applied. FAIMS-MS analyses were performed by direct infusion, via a syringe pump (11-plus, Harvard Apparatus, Cambridge, UK), operated at a rate of $10 \mu \mathrm{L} \mathrm{min}{ }^{-1}$.

HPLC separation for LC-FAIMS-MS and LC-MS was carried out on an Agilent Poroshell 120 hydrophilic interaction liquid chromatography (HILIC) column (4.6 x $50 \mathrm{~mm}, 2.7 \mu \mathrm{m}$ ), maintained at $35^{\circ} \mathrm{C}$, with a $5 \mathrm{~mm}$ HILIC guard column (Agilent Technologies, Santa Clara, USA) and a $5 \mu \mathrm{L}$ sample injection volume (autosampler maintained at $6^{\circ} \mathrm{C}$ ). A gradient chromatographic method utilized mobile phase $A$ consisting of $\mathrm{H}_{2} \mathrm{O}$ with $10 \mathrm{mM}$ ammonium acetate and $0.1 \%$ acetic acid and mobile phase $\mathrm{B}$ consisting of $98: 2 \mathrm{v} / \mathrm{v} \mathrm{ACN}: \mathrm{H}_{2} \mathrm{O}$ with $10 \mathrm{mM}$ ammonium acetate and $0.1 \%$ acetic acid. The chromatographic method was as follows: hold at $100 \% \mathrm{~B}$ from 0-0.5 min, decrease to $92 \% \mathrm{~B}$ at $1.5 \mathrm{~min}$, decrease further to $82 \% \mathrm{~B}$ at $2.5 \mathrm{~min}$ and hold for $1 \mathrm{~min}$, before increasing back up to 100\% B at 6 min and holding for 2 min, for a total run time of $8 \mathrm{~min}$ and operated at a flow rate of $0.275 \mathrm{~mL} \mathrm{~min}^{-1}$. A solution of $205.1 \mu \mathrm{g} \mathrm{mL}-1$ sodium acetate (in 90:10 v/v ACN: ${ }_{2} \mathrm{O}$ ) was infused into the LC mobile phase post-column at a flow rate of $5 \mu \mathrm{L} \mathrm{min}$ m $^{-1}$ in order to promote the formation of doubly sodiated adducts of the steroid metabolites in the ESI source (post LC separation, prior to FAIMS and MS).

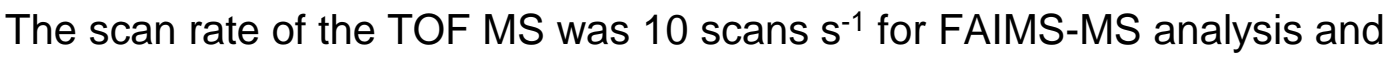
2 scans $\mathrm{s}^{-1}$ for LC-FAIMS-MS and LC-MS analysis in the $\mathrm{m} / \mathrm{z}$ range 80-1500 Da. The MS nebulizer pressure was set to 50 psig with a sheath gas $\left(N_{2}\right)$ flow of $11 \mathrm{~L} \mathrm{~min}^{-1}$ at $250{ }^{\circ} \mathrm{C}$, with a nozzle voltage of $2000 \mathrm{~V}$. The capillary voltage was set to $3500 \mathrm{~V}$, the fragmentor voltage was set to $200 \mathrm{~V}$ and the drying gas $\left(\mathrm{N}_{2}\right)$ flow set to $7 \mathrm{~L} \mathrm{~min}^{-1}$ at $150{ }^{\circ} \mathrm{C}$, which also provides $\mathrm{N}_{2}$ as the carrier gas in the FAIMS device. FAIMS-MS analyses were performed using a two-dimensional FAIMS scan (or sweep) in the DF range from 180-300 $\mathrm{Td}$ (in steps of $10 \mathrm{Td}$ ) and CF range from -2-5 Td (in steps of $0.05 \mathrm{Td}$ ). The FAIMS parameters were optimized for the seven metabolites and LCFAIMS-MS analyses were performed at three conditions (each at DF $260 \mathrm{Td}$ ) of CF 1.35 Td for DHEAS, TS and TG, CF 2.05 Td for ETS, ETG and ECG, and CF 2.70 $\mathrm{Td}$ for $\mathrm{ADG}$ (where $1 \mathrm{Td}=10^{-17} \mathrm{~V} \mathrm{~cm}^{2}$ ). The data were acquired in three analysis runs with the FAIMS at held static at one condition per run, however it is possible to acquire the data in one analysis by alternating between the FAIMS conditions, to allow for the determination of all seven steroid metabolites in a single run. Data were 
acquired using Agilent MassHunter Acquisition B.05.00 and prototype FAIMS Control software, and processed using Agilent MassHunter Qualitative Analysis B.05.00. Microsoft Excel and OriginLab 2015 (Academic version b9.2.272).

\section{Results and Discussion}

\section{FAIMS-MS investigation of steroid metabolites}

Preliminary FAIMS-MS analysis of TG/ETG showed no significant separation of the deprotonated isobaric steroid metabolites in negative ion mode (data not shown). However, there was a partial resolution observed in positive ion mode for the protonated and sodiated adducts. Therefore, a FAIMS-MS investigation into the separation of the seven steroid metabolites was conducted in positive ion mode to study the effects of different cations $\left(\mathrm{H}^{+}, \mathrm{NH}_{4}^{+}, \mathrm{Na}^{+}, \mathrm{K}^{+}, \mathrm{Cs}^{+}\right)$on the FAIMS separation. Figure 2 is a summary of the variation of CF with the applied DF for the cationized steroid metabolites following direct infusion into the FAIMS-MS, where the CF values plotted are for the apex of the FAIMS peak at each DF; note that not all of the adduct ions were observed for each of the steroids. The FAIMS characteristics of the steroid ions result from differences between the complex non-linear relationship for ion mobility and field strength at high fields, compared to the field-independent ion mobility under low field conditions. Differential ion mobility is influenced by cationisation, so the appropriate choice of cation may result in the separation of isobaric ions where the protonated and deprotonated molecules are not resolved. $8,20,21$

Separation of TG/ETG (Figure 2, dark blue/green) was observed for the protonated, sodiated and cesium adducts, with the best separation obtained for the doubly sodiated adducts, $[\mathrm{TG}+2 \mathrm{Na}-\mathrm{H}]^{+}$and $[\mathrm{ETG}+2 \mathrm{Na}-\mathrm{H}]^{+}$. Separation of ADG/ECG (Figure 2 , red/grey) was only achieved for the sodiated and doubly sodiated adducts. The $[\mathrm{ADG}+\mathrm{Na}]^{+}$adduct was transmitted at lower $\mathrm{CF}$ values compared to $[\mathrm{ECG}+\mathrm{Na}]^{+}$for all DFs, but for the doubly sodiated adducts the order is reversed with the $[\mathrm{ADG}+2 \mathrm{Na}-\mathrm{H}]^{+}$adduct observed at higher $\mathrm{CFs}$ than $[\mathrm{ECG}+2 \mathrm{Na}-\mathrm{H}]^{+}$. This results in better resolution of the doubly sodiated adducts in comparison to the singly sodiated ions, with peak widths of $~ 1 \mathrm{Td}$ allowing the ions to be transmitted at $2.05 \mathrm{Td}$ (ECG) and 2.70 Td (ADG) without overlap. The sulfated steroid metabolites were generally detected as di-cations, except for the protonated forms of TS/ETS, which showed no separation (Figure 2, purple/light blue), and only small differences between the separation with the different metal cations were observed. In each case, the ETS (Figure 2, light blue) was separated from TS/DHEAS (Figure 2, purple/orange), with the greatest resolution observed for the $[\mathrm{M}+\mathrm{Na}+\mathrm{K}-\mathrm{H}]^{+}$adducts. TS/DHEAS were not separated by FAIMS for any combination of cations.

The doubly sodiated adducts $\left([\mathrm{M}+2 \mathrm{Na}-\mathrm{H}]^{+}\right)$were selected for the LC-FAIMS-MS analysis, because of the separation of the steroid isobars, with post-column addition 
of sodium acetate to promote the formation of sodiated adducts. A sodium concentration of $0.20 \mathrm{mg} \mathrm{mL}^{-1}$ provided the optimum sensitivity for the $[\mathrm{M}+2 \mathrm{Na}-\mathrm{H}]^{+}$ ions, suppressing other adducts without promoting the formation of higher sodiated clusters. For the combination of FAIMS with LC-MS, the FAIMS DF and CF separations of the doubly sodiated adducts were further investigated and optimized (Figure 3 (a)). As a compromise between separation and signal intensity, DF 260 Td was chosen from the FAIMS-MS analysis (Figure $3(b)$ ) to be used for all subsequent LC-FAIMS-MS analyses. Three optimum FAIMS CFs were selected (Figure 3 (b)) to transmit the steroid metabolites $\left([\mathrm{M}+2 \mathrm{Na}-\mathrm{H}]^{+}\right)$: CF 1.35 Td for DHEAS, TS and TG; CF 2.05 Td for ETS, ETG and ECG; and CF 2.70 Td for ADG. This choice of FAIMS settings allowed the selective transmission of each of the glucuronide isobars in a mixture of the seven steroid standards. DHEAS and TS were not separated by FAIMS (same CF for transmission) or MS (same $m / z$ ), so it was necessary to achieve chromatographic separation of the DHEAS and TS in the LC-FAIMS-MS analysis. The relationship between the structures of steroid ions and their low field ion mobility has been reported for the separation of TG/ETG using TWIMS, and the resolution of the sodiated dimers of androsterone epimers by static field DTIMS. ${ }^{7,22}$ However, relating structure to CF in FAIMS is more complicated due to the nonlinear relationship between ion mobility and field strength at high fields. This is dependent upon several factors including the effective temperature (determined by the carrier gas temperature and frictional heating), interactions between the ion and buffer gas and structural changes as the field is increased. Therefore modelling the CF values for these steroid metabolites was not attempted in this study.

\section{Determination of steroid metabolites using LC-FAIMS-MS}

Reversed-phase (C18) and HILIC chromatography were both investigated for the separation of the steroid metabolites. The glucuronides were chromatographically resolved using a reversed phase method, however DHEAS and TS were not resolved. The glucuronides were also separated by FAIMS, but DHEAS and TS were not, so the only combination found to be capable of separating all seven metabolites was HILIC-FAIMS-MS. A fast HILIC method (8 min) was selected due to the rapid elution of the three steroid sulfates (Figure $4(\mathrm{a})$ ), where TS/ETS co-elute ( $m / z$ 413.14, retention times 2.20/2.22 min respectively), but are chromatographically separated from DHEAS ( $\mathrm{m} / \mathrm{z}$ 413.14, retention time $1.93 \mathrm{~min}$ ), as shown in Figure 4 (a) (FAIMS off). As TS/ETS are separated by CF in the FAIMS, the combination of LC-FAIMS-MS allows separation of each of the steroid sulfates (Figure 4 (a), CFs 1.35 Td and 2.05 Td). In LC-MS (Figure 4, FAIMS off) the glucuronides TG/ETG ( $\mathrm{m} / \mathrm{z}$ 509.21, 7.23/7.09 min respectively) and ADG/ECG (m/z 511.23, 6.99/7.07 min respectively) co-elute, as shown in Figure 4 (b) and (c) respectively; therefore all of the steroid metabolites could not be identified and quantified using LC-MS alone. TG/ETG and ADG/ECG are selectively transmitted using the FAIMS at CFs 1.35 Td and 2.05 Td for TG/ETG (Figure 4 (b)) and CFs 2.05 Td and 2.70 Td for ECG/ADG 
(Figure 4 (c)). LC-FAIMS-MS allows a high level of discrimination (>95\%) for the steroid metabolites spiked into urine (Table 1) where discrimination was calculated from the peak areas of the spiked standards $\left(1 \mu \mathrm{g} \mathrm{mL}^{-1}\right)$ run individually and as a mixture using LC-FAIMS-MS at the three chosen CFS (DF 260 Td). Intra-day reproducibility of LC-FAIMS-MS of spiked standards $\left(1 \mu \mathrm{g} \mathrm{mL}^{-1}\right)$ was found to be comparable to LC-MS (\%RSD <10\%) with no internal standard used, as shown in Table 1 (note that for the LC-MS of TS/ETS and ADG/ECG, individual \%RSDs could not be determined due to co-elution). Therefore, a three-dimensional separation of all seven of the targeted steroid metabolites was achieved through the incorporation of FAIMS with LC-MS with an analysis time of $<10$ minutes (Figure 5).

The addition of sodium acetate to promote the formation of the doubly sodiated adducts of the steroid metabolites also results in the formation of singly charged clusters of the $[\mathrm{M}+2 \mathrm{Na}-\mathrm{H}]^{+}$adducts with sodium acetate of the formula $[\mathrm{M}+2 \mathrm{Na}-$ $\mathrm{H}]^{+}+(\mathrm{NaOAc}) \mathrm{n}$. These were observed in the mass spectra in MS and FAIMS-MS modes, and the mass spectra extracted from the chromatographic peak retention times for each steroid in LC-MS and LC-FAIMS-MS. Using MS alone, each of the steroid metabolites forms clusters with the sodium acetate ranging from one sodium acetate up to a cluster of thirteen (Supplementary Figures 1-3 (a)). In FAIMS-MS, different cluster patterns are observed for both the sulfates (Supplementary Figure 1 (b)) and the glucuronides (Supplementary Figures 2 \& 3 (b)). Further differences in the cluster pattern intensities can be observed in LC-FAIMS-MS (Supplementary Figures 1-3 (c \& d)) in comparison to FAIMS-MS. The different cluster patterns observed for each steroid metabolite arise from the transmission of the clusters through the FAIMS device. For example, at the CF for transmission of ADG (CF 2.70 Td, DF 260 Td) in the LC-FAIMS-MS (Supplementary Figure 3 (c \& d)) none of the cluster ions with sodium acetate are observed as they all traverse the device at lower $\mathrm{CF}$ values, therefore the only ion observed is the doubly sodiated ion of ADG $\left([\mathrm{ADG}+2 \mathrm{Na}-\mathrm{H}]^{+}\right)$. However, the lower-ordered clusters for ECG are transmitted through the device at CF 2.05 Td (DF 260 Td). The cluster patterns of each of the sulfate ions in the LC-FAIMS-MS (Supplementary Figure 1 (c \& d)) are highly diverse with clusters for DHEAS observed across the mass spectrum, higher ordered clusters for TS observed and lower ordered clusters for ETS observed; therefore, creating unique cluster patterns at the CFs for transmission of each ion. In tandem MS, a fragmentation pattern can be used to confirm the identity of species. ${ }^{23,24}$ Here we propose that using FAIMS, cluster patterns could be used as an additional qualifying identification tool to confirm the identity of each of the steroid metabolites utilizing the FAIMS pre-selection, either by FAIMS-MS or LC-FAIMS-MS.

Table 2 shows the quantitative characteristics of the LC-FAIMS-MS method for the determination of standards of the seven steroids. Good linearity $\left(R^{2}>0.99\right)$ was observed in a six point calibration across the range $31-1000 \mathrm{ng} \mathrm{mL}^{-1}$ for each of the targeted doubly sodiated ions $\left([\mathrm{M}+2 \mathrm{Na}-\mathrm{H}]^{+}\right)$of the steroid metabolites. [ADD TEXT HERE] Limits of detection (LOD, S:N 3:1) were in the range 1-6 $\mathrm{ng} \mathrm{mL}^{-1}$ and limits of 
quantification (LOQ, S:N 10:1) in the range 3-20 ng mL-1. Internal standards were not used in not used in this LC-FAIMS-MS method development, but could be incorporated in routine assays. These data demonstrate the stability of the miniaturized FAIMS and ion source configuration used in this study, although the CFs for ion transmission would require optimization for other FAIMS chip and ion source configurations. Using LC-MS alone without the FAIMS, some of the steroid metabolites could not be quantified using this chromatographic method without a significantly increased chromatographic run time due to the co-elution of the isobaric steroid metabolites.

LC-FAIMS-MS in comparison to LC-MS has been shown previously to increase linear dynamic ranges and lower LODs and LOQs due to $\mathrm{S}: \mathrm{N}$ improvements of components of interest resulting from a reduction in chemical noise ${ }^{25}$ This was demonstrated for the targeted steroid metabolites investigation in unspiked human urine. Figure 6 demonstrates the determination of ADG/ECG in urine by LC-MS and LC-FAIMS-MS. Using LC-FAIMS-MS the two isobars are individually identified and S:N improvements of $>250 \%$ are observed (ADG S:N 1450, ECG S:N 4510) compared to the unresolved ADG/ECG peaks in LC-MS (S:N 180). Increases in the $\mathrm{S}: \mathrm{N}$ for components using LC-FAIMS-MS is advantageous as they can lead to lower LODs and LOQs, enhancing the response of targeted components by reducing noise and removing isobaric interferences from the matrix.

\section{Conclusions}

The combination of a miniaturized FAIMS separation with LC-MS using a HILIC column has enabled the determination of seven anabolic steroid metabolites in human urine. A short chromatographic run time (8 $\mathrm{min}$ ) was made possible by FAIMS separation of co-eluting isobaric steroid metabolites. A reduction in chemical noise from the urine matrix and improved $\mathrm{S}: \mathrm{N}$ is also observed. The LC-FAIMS-MS method showed good qualitative and quantitative responses for the steroid metabolites, with an additional identification tool using cluster patterns made possible using FAIMS. Higher sensitivity for the naturally low-abundant steroid metabolites could be achieved by combining the LC-FAIMS-MS with tandem mass spectrometry, for example a triple quadrupole mass spectrometer.

\section{Acknowledgments}

The authors thank Owlstone Ltd. and Loughborough University for financial support, and Owlstone Ltd. and Agilent Technologies for the provision of instrumentation. 


\section{References}

[1] Jeanneret, F.; Tonoli, D.; Rossier, M.; Saugy, M.; Boccard, J.; Rudaz, S.; J. Chromatogr. A, 2016, 1430, 97-112.

[2] WADA Technical Document TD2016EAAS (current version), 2016, https://www.wada-ama.org/en/resources/science-medicine/td2016-eaas

[3] Van Renterghem, P.; Van Eenoo, P.; Geyer, H.; Schanzer, W.; Delbeke, F.; Steroids, 2010, 75, 154-163.

[4] Yamashita, K.; Okuyama, M.; Nakagawa, R.; Honma, S.; Satoh, F.; Morimoto, R.; Ito, S.; Takahashi, M.; Numazawa, M.; J. Chromatogr. A, 2008, 1200, 114121.

[5] Borts, D.; Bowers, L.; J. Mass Spectrom., 2000, 35, 50-61.

[6] Kushnir, M.; Rockwood, A.; Roberts, W.; Pattison, E.; Owen, W.; Bunker, A.; Meikle, A.; Clin. Chem., 2006, 52, 1559-1567.

[7] Kaur-Atwal, G.; Reynolds, J.; Mussell, C.; Champarnaud, E.; Knapman, T.; Ashcroft, A.; O'Connor, G.; Christie, S.; Creaser, C.; Analyst, 2011, 136, 39113916.

[8] Chouinard, C.; Beekman, C.; Kemperman, R.; King, H.; Yost, R.; Int. J. Ion Mobil. Spec., 2017, 20, 31-39.

[9] Srebalus Barnes, C.; Hilderbrand, A.; Valentine, S.; Clemmer, D.; Anal. Chem., 2002, 74, 26-36.

[10] Paglia, G.; Kliman, M.; Claude, E.; Geromanos, S.; Astarita, G.; Anal. Bioanal. Chem., 2015, 407, 4995-5007.

[11] Zhu, M.; Bendiak, B.; Clowers, B.; Hill, H.; Anal. Bioanal. Chem., 2009, 394, 1853-1867.

[12] Ahonen, L.; Fasciotti, M.; af Gennas, G.; Kotiaho, T.; Daroda, R.; Eberlin, M.; Kostiainen, R.; J. Chromatogr. A, 2013, 1310, 133-137.

[13] Devenport, N.; Reynolds, J.; Parkash, V.; Cook, J.; Weston, D.; Creaser, C.; J. Chromatogr. B, 2011, 879, 3797-3801.

[14] Ray, J.; Kushnir, M.; Yost, R.; Rockwood, A.; Meikle, A.; Clin. Chim. Acta, 2015, 438, 330-336.

[15] Guddat, S.; Thevis, M.; Kapron, J.; Thomas, A.; Schanzer, W.; Drug Test. 
Analysis, 2009, 1, 545-553.

[16] Jin, W.; Jarvis, M.; Star-Weinstock, M.; Altemus, M.; Anal Bioanal Chem, 2013, 405, 9497-9508.

[17] WADA Prohibited List 2016, 2015, https://www.wadaama.org/en/resources/science-medicine/prohibited-list. [Accessed 04 April 2016].

[18] Brown, L.; Smith, R.; Toutoungi, D.; Reynolds, J.; Bristow, A.; Ray, A.; Sage, A.; Wilson, I.; Weston, D.; Boyle, B.; Creaser, C.; Anal. Chem., 2012, 84, 4095-4103.

[19] Shvartsburg, A.; Tang, K.; Smith, R.; Holden, M.; Rush, M.; Thompson, A.; Toutoungi, D.; Anal. Chem., 2009, 81, 8048-8053.

[20] Clowers, B.; Hill, H.; J. Mass Spectrom., 2006, 41, 339-351.

[21] Domalain, V.; Tognetti, V.; Hubert-Roux, M.; Lange, C.; Joubert, L.; Baudoux, J.; Rouden, J.; Afonso, C.; J. Am. Soc. Mass Spectrom., 2013, 24, 1437-1445.

[22] Chouinard, C.; Cruzeiro, V.; Roitberg, A.; Yost, R.; J. Am. Soc. Mass Spectrom., 2017, 28, 323-331.

[23] Holcapek, M.; Kolarova, L.; Nobilis, M.; Anal. Bioanal. Chem., 2008, 391, 5978.

[24] Bowen, B.; Northen, T.; J. Am. Soc. Mass Spectrom., 2010, 21, 1471-1476.

[25] Smith, R.; Toutoungi, D.; Reynolds, J.; Bristow, A.; Ray, A.; Sage, A.; Wilson, I.; Weston, D.; Boyle, B.; Creaser, C.; J. Chromatogr. A, 2013, 1278, 76-81. 
Figures

(a)

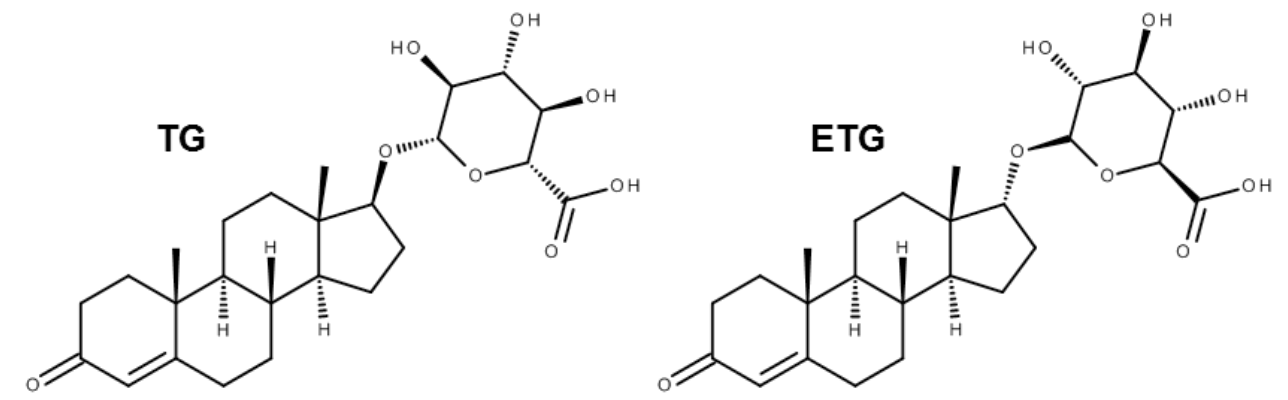

(b)

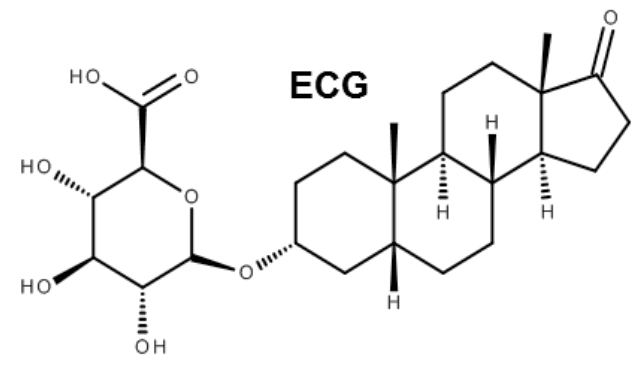

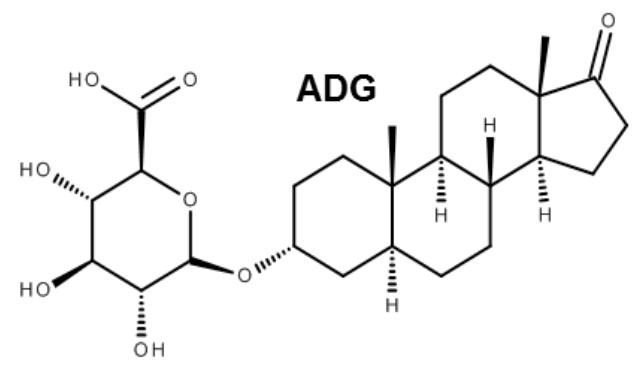

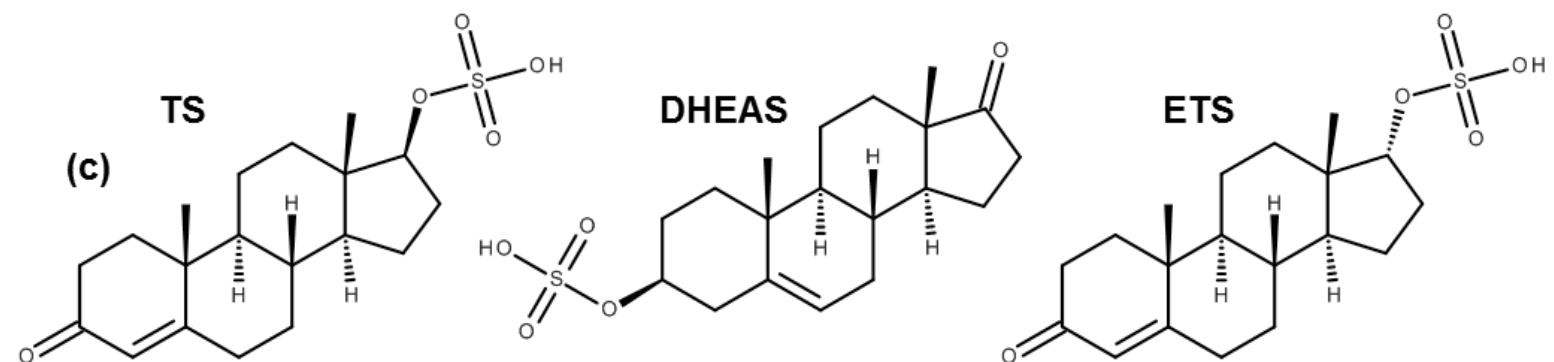

Figure 1: Structures of the targeted steroid metabolites (a) testosterone and epitestosterone glucuronides (TG/ETG), (b) etiocholanolone and androsterone glucuronides (ECG/ADG), and (c) testosterone, dehydroepiandrosterone and epitestosterone sulfates (TS, DHEAS and ETS) 
$[\mathrm{M}+\mathrm{H}]+$

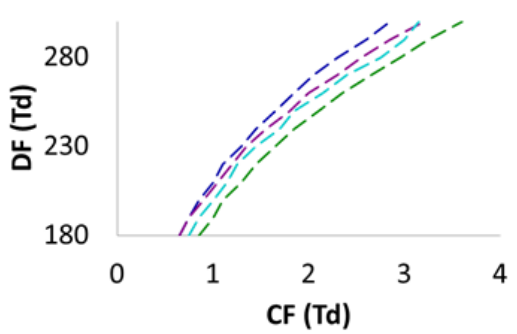

$[\mathrm{M}+\mathrm{K}]^{+}$

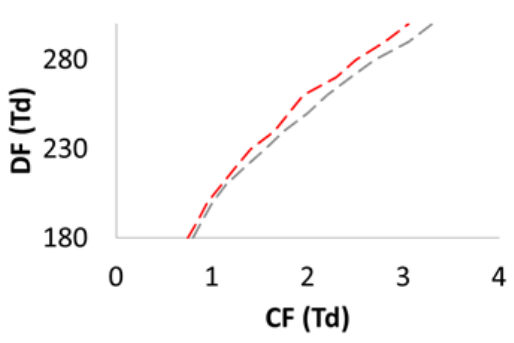

$[\mathrm{M}+\mathrm{Cs}]^{+}$

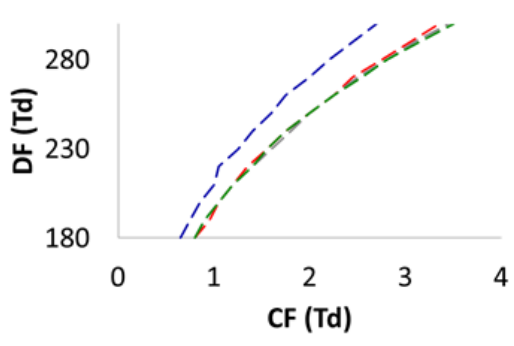

$[\mathrm{M}+\mathrm{NH} 4]^{+}$

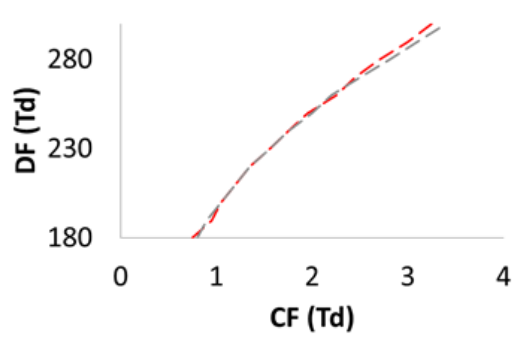

$[\mathrm{M}+2 \mathrm{Na}-\mathrm{H}]^{+}$

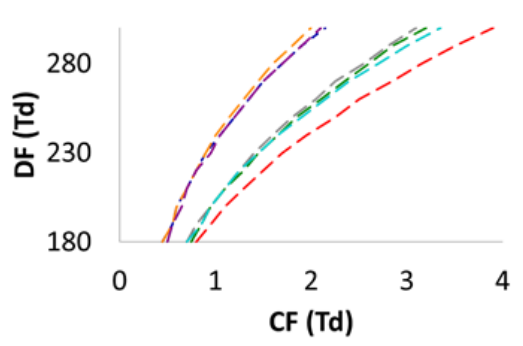

$[\mathrm{M}+\mathrm{Cs}+\mathrm{Na}-\mathrm{H}]^{+}$

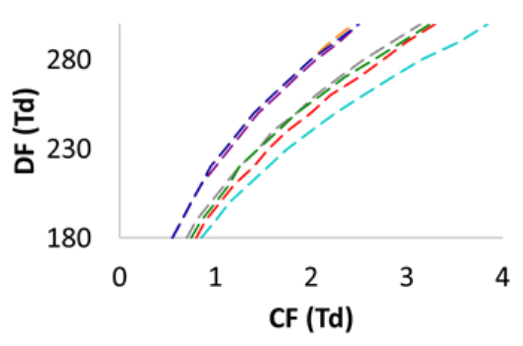

$[\mathrm{M}+\mathrm{Na}]^{+}$

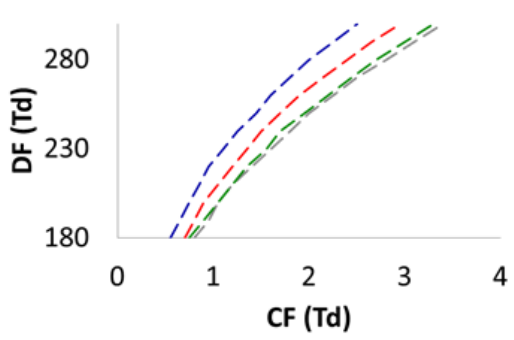

$[\mathrm{M}+\mathrm{Na}+\mathrm{K}-\mathrm{H}]^{+}$

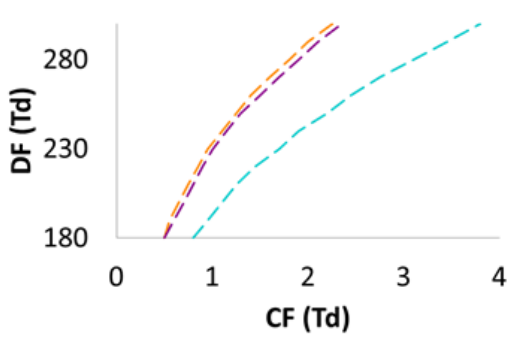

$[\mathrm{M}+2 \mathrm{CS}-\mathrm{H}]^{+}$

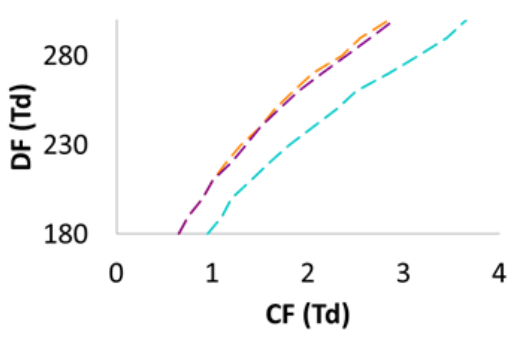

Figure 2: FAIMS-MS analysis of steroid metabolite adducts; plotted are the CF values for the apex of each FAIMS peak signal intensity for each DF (10 Td steps) for ADG (red), DHEAS (orange), ECG (grey), ETG (green), ETS (light blue), TG (dark blue) and TS (purple) 

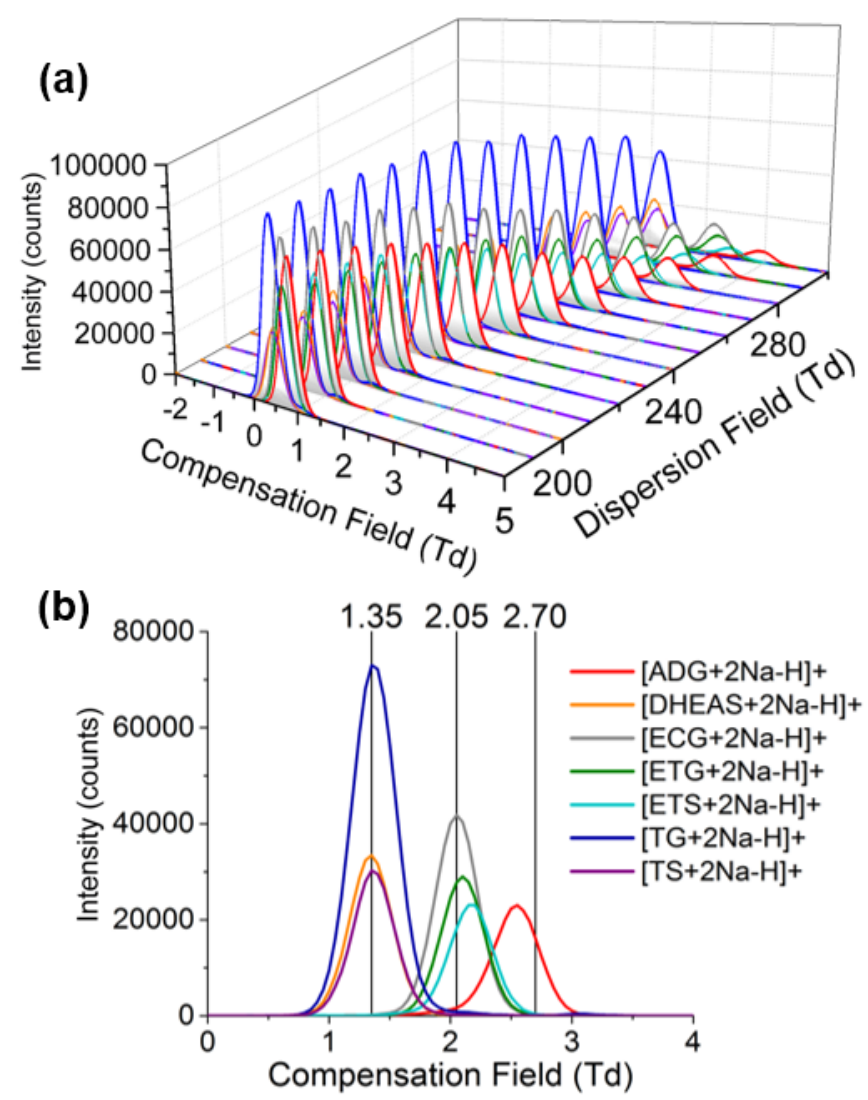

Figure 3: (a) FAIMS-MS DF vs. CF waterfall plot for the seven targeted steroid metabolites in 98:2 v/v ACN: $\mathrm{H}_{2} \mathrm{O}$ with $10 \mathrm{mM}$ ammonium acetate and $0.1 \%$ acetic acid; (b) CF plot at DF $260 \mathrm{Td}$ with three optimized CFs $1.35 \mathrm{Td}, 2.05 \mathrm{Td}$ and $2.70 \mathrm{Td}$ 


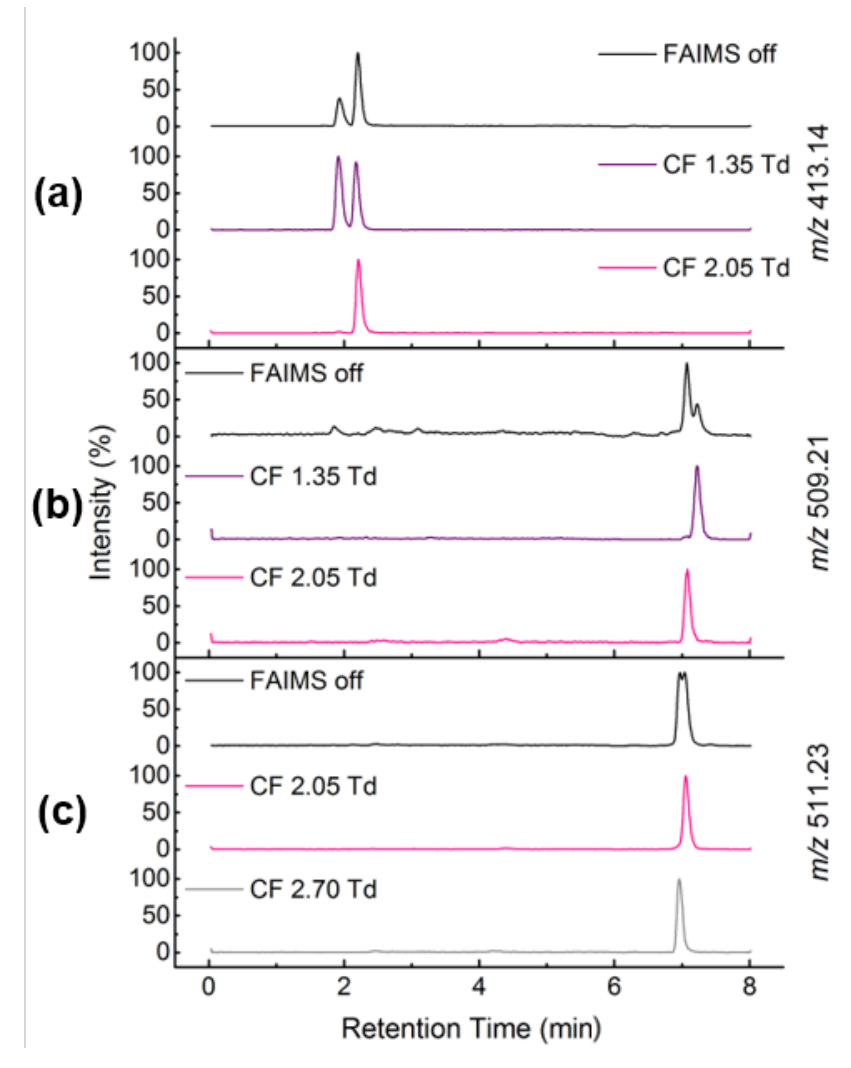

Figure 4: Mass extracted ion chromatograms for the seven steroids spiked $\left(1 \mu \mathrm{g} \mathrm{mL}^{-1}\right)$ into urine with LC-MS (FAIMS off) shown in black and LC-FAIMS-MS chromatograms (at DF 260 Td) with the optimum CFs chosen for each steroid: CF 1.35 Td (purple) for DHEAS (a) and TS (a) ( $m / z$ 413.14) and TG (b) ( $m / z$ 509.21); CF 2.05 Td (pink) for ETS (a) ( $m / z$ 413.14), ETG (b) (509.21) and ECG (c) ( $\mathrm{m} / z$ 511.23); and CF 2.70 Td (grey) for ADG (c) ( $\mathrm{m} / \mathrm{z} 511.23)$ 


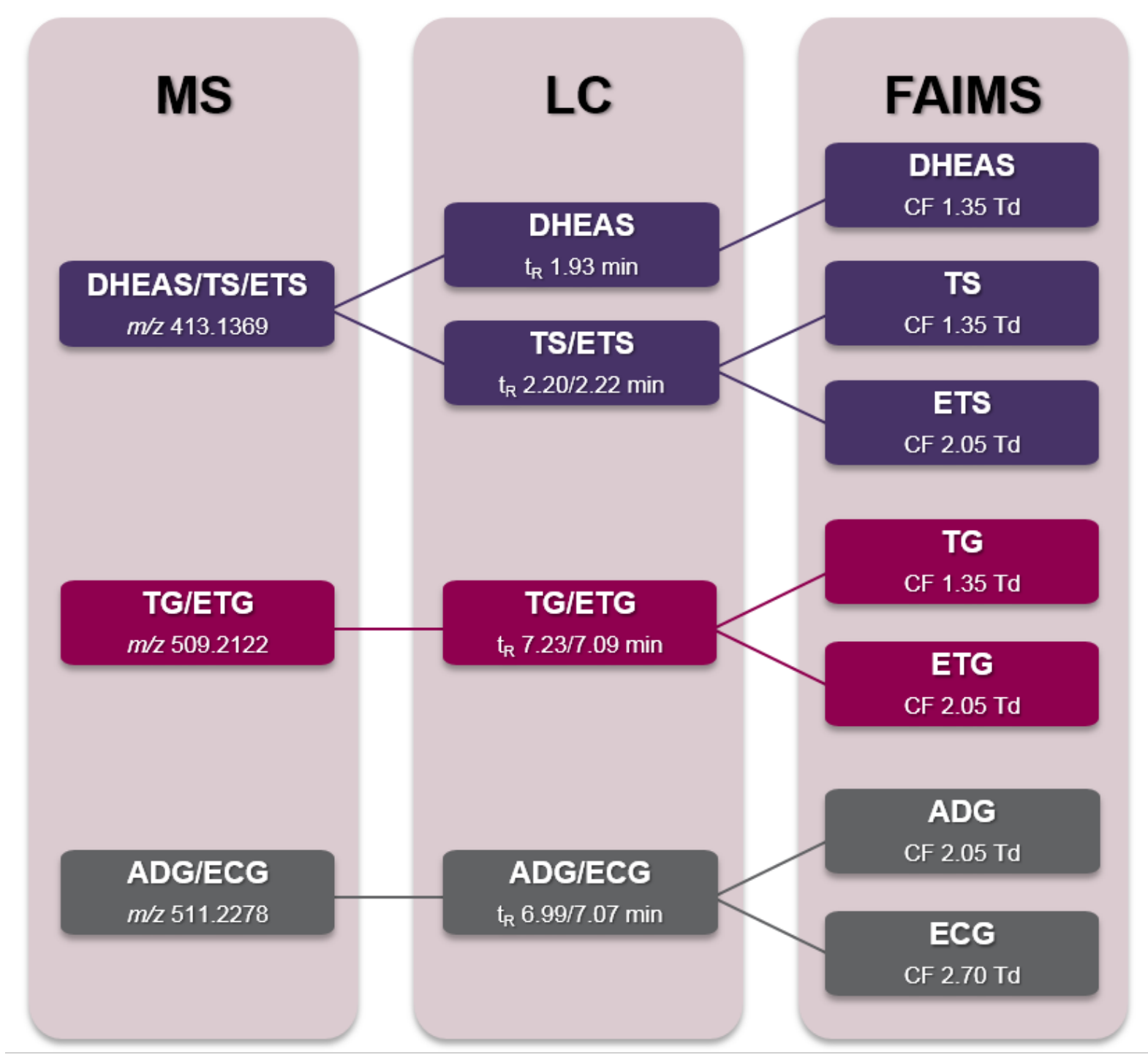

Figure 5: Summary of the three-dimensional separation achieved for seven steroid metabolites using LC-FAIMS-MS, where isobaric steroids are color coded: $\mathrm{m} / \mathrm{z} 413.14$ (purple), $m / z 509.21$ (pink), and $m / z 511.23$ (grey) 
Table 1: Discrimination (\%) between steroid metabolites, calculated as [Area individual/Areamixed] $\times 100$, and relative standard deviations (\%) for LC-MS and LC-FAIMSMS analysis of spiked urine replicate aliquots $(n=6)$

\begin{tabular}{cccc}
$\begin{array}{c}\text { Steroid } \\
\left([\mathrm{M+2Na-H}]^{+}\right)\end{array}$ & Discrimination (\%) & $\begin{array}{c}\text { Relative standard deviation }(\%, \mathbf{n}=\mathbf{6}) \\
\text { LC-MS }\end{array}$ & $\begin{array}{c}\text { LC-FAIMS-MS } \\
\text { DHEAS }\end{array}$ \\
TS & 97.0 & 5.6 & 4.3 \\
ETS & 99.5 & 4.9 & 5.1 \\
ADG & 96.2 & & 2.8 \\
ECG & 95.1 & 12.8 & 5.8 \\
ETG & 97.2 & 12.2 & 3.6 \\
TG & 96.0 & 8.7 & 4.9 \\
& 95.1 & & 9.1 \\
\hline
\end{tabular}

Table 2: Summary of the quantitative responses for the steroid metabolites using LC-FAIMSMS

$\begin{array}{cccc}\text { Steroid } & \begin{array}{c}\text { LOD } \\ \left(\mathbf{n g ~} \mathbf{~ m}^{-1}\right)\end{array} & \begin{array}{c}\text { LOQ } \\ \left(\mathbf{n g ~} \mathbf{~ m L}^{-1}\right)\end{array} & \begin{array}{c}\mathbf{R}^{2} \\ \text { Linear }\end{array} \\ \text { DHEAS } & 1.1 & 3.8 & 0.9975 \\ \text { TS } & 1.3 & 4.2 & 0.9955 \\ \text { ETS } & 1.1 & 3.7 & 0.9919 \\ \text { ADG } & 6.1 & 20.3 & 0.9900 \\ \text { ECG } & 2.8 & 9.3 & 0.9927 \\ \text { ETG } & 3.7 & 12.3 & 0.9927 \\ \text { TG } & 1.0 & 3.4 & 0.9926\end{array}$




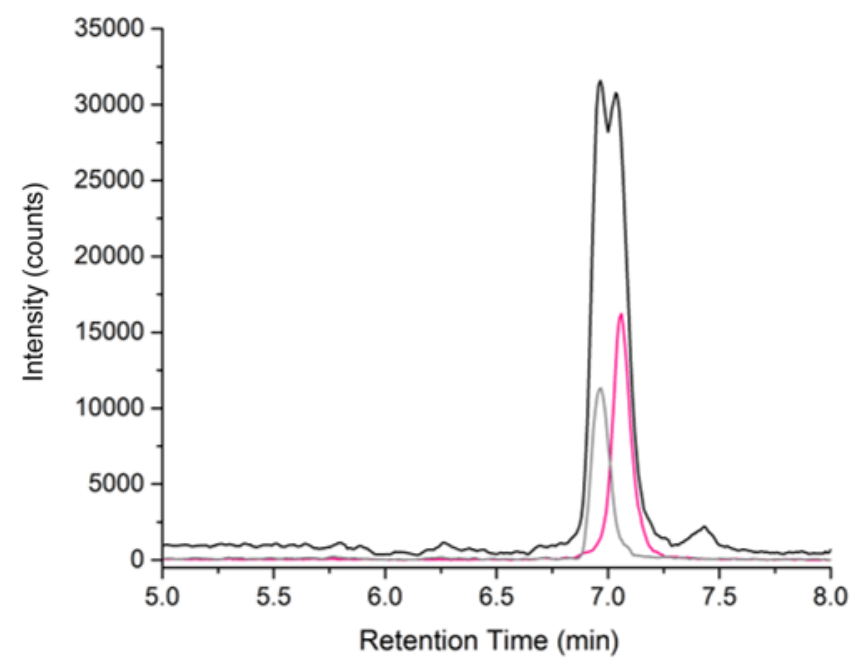

Figure 6: Extracted ion chromatographs ( $\mathrm{m} / \mathrm{z}$ 511.23, DF $260 \mathrm{Td}$ ) for unspiked urine with LCMS (black) and LC-FAIMS-MS at CF 2.05 Td (pink, ECG) and at CF 2.70 Td (grey, ADG). S: $N$ improvement of $>250 \%$ are observed 


\section{LC-FAIMS-MS Steroids}

Rapid analysis of anabolic steroid metabolites in urine by combining field asymmetric waveform ion mobility spectrometry with liquid chromatography and mass spectrometry

Kayleigh L. Arthur ${ }^{1}$, Matthew A. Turner ${ }^{1}$, Alan D. Brailsford ${ }^{2}$, Andrew T. Kicman², David A. Cowan ${ }^{2}$, James C. Reynolds ${ }^{1 \star}$, Colin S. Creaser ${ }^{1 *}$

${ }^{1}$ Centre for Analytical Science, Department of Chemistry, Loughborough University, Loughborough, Leicestershire, LE11 3TU, UK

2 Drug Control Centre, King's College London, London, SE1 9NH, UK

Supplementary information 
(a)

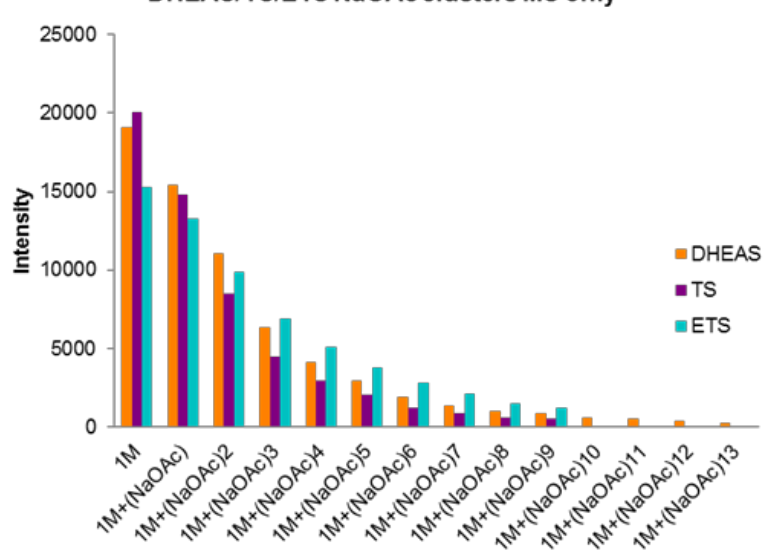

(c)

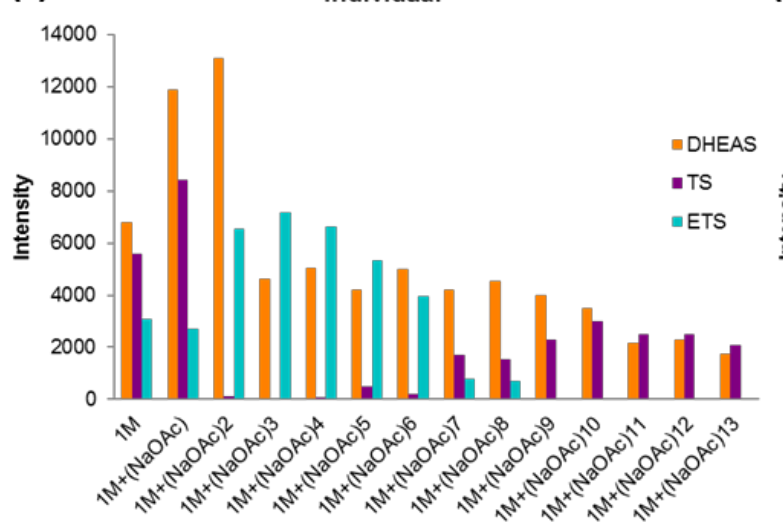

(b) DHEAS/TS/ETS NaOAc clusters FAIMS-MS

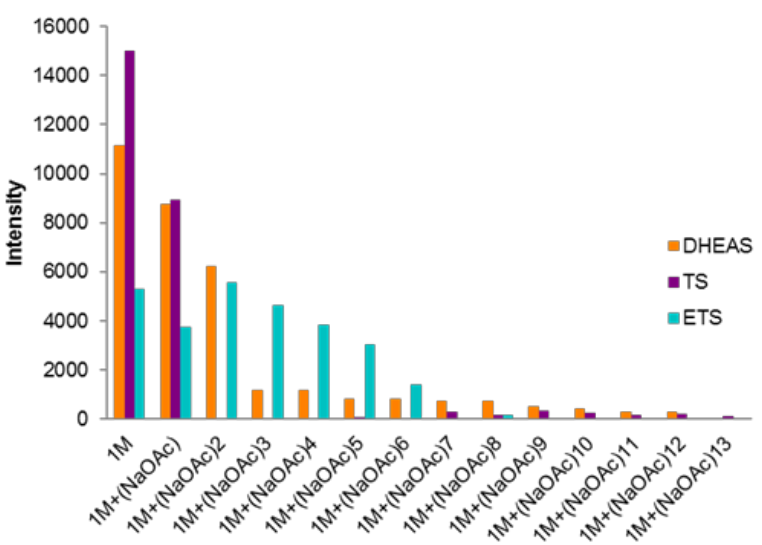

(d)

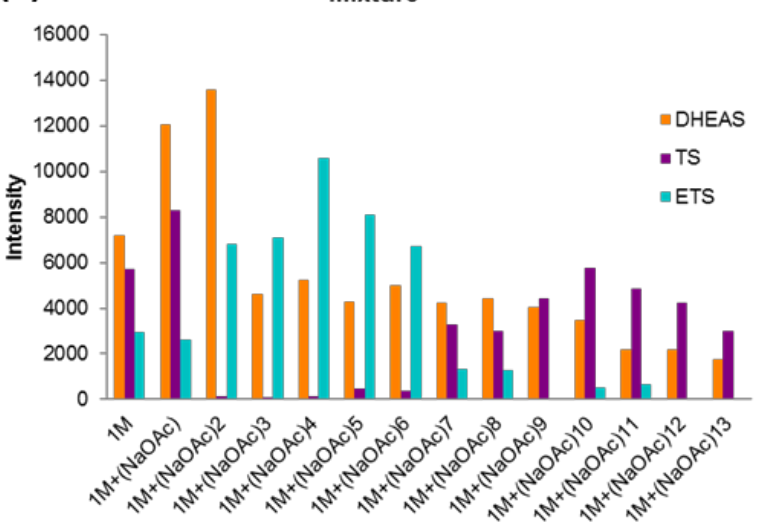

Supplementary Figure 1: Cluster patterns observed in the mass spectra for the doubly sodiated $[\mathrm{M}+2 \mathrm{Na}-\mathrm{H}]^{+}$ions of DHEAS/TS/ETS ( $\mathrm{m} / \mathrm{z}$ 413.137) analyzed individually using (a) MS, (b) FAIMS-MS (DF 260 Td, CF 1.35 Td (DHEAS/TS) and CF 2.05 Td (ETS)) and (c) LCFAIMS-MS; (d) LC-FAIMS-MS for DHEAS/TS/ETS analyzed as a mixture 
(a)

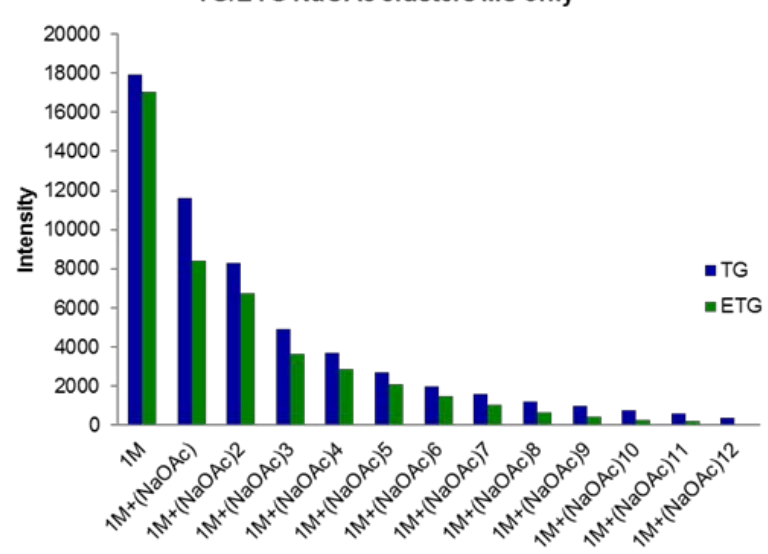

(c) TG/ETG NaOAc clusters LC-FAIMS-MS individual

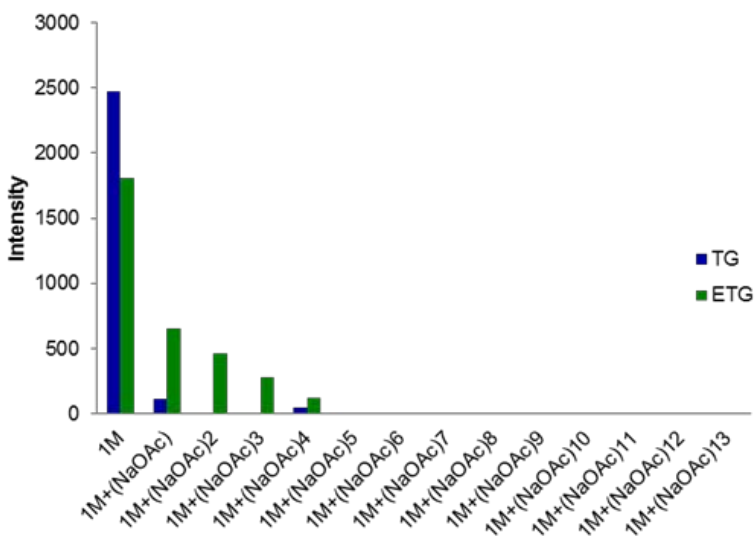

(b) TG/ETG NaOAc clusters FAIMS-MS

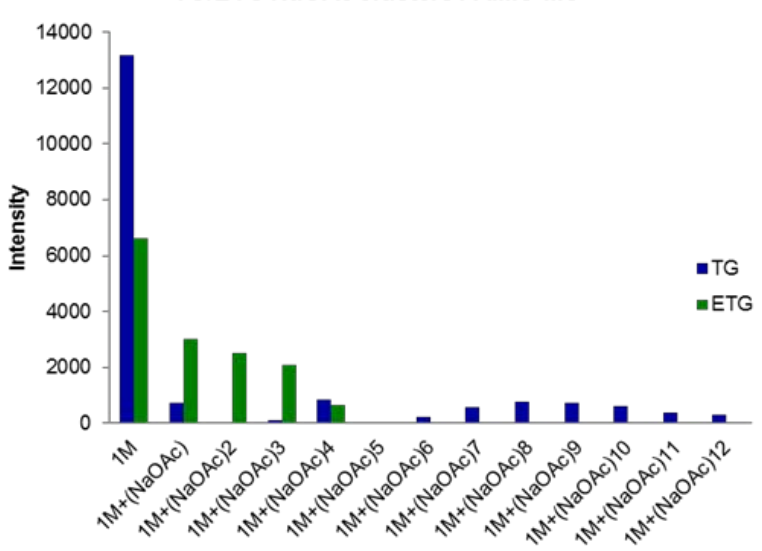

(d) TG/ETG NaOAc clusters LC-FAIMS-MS mixture

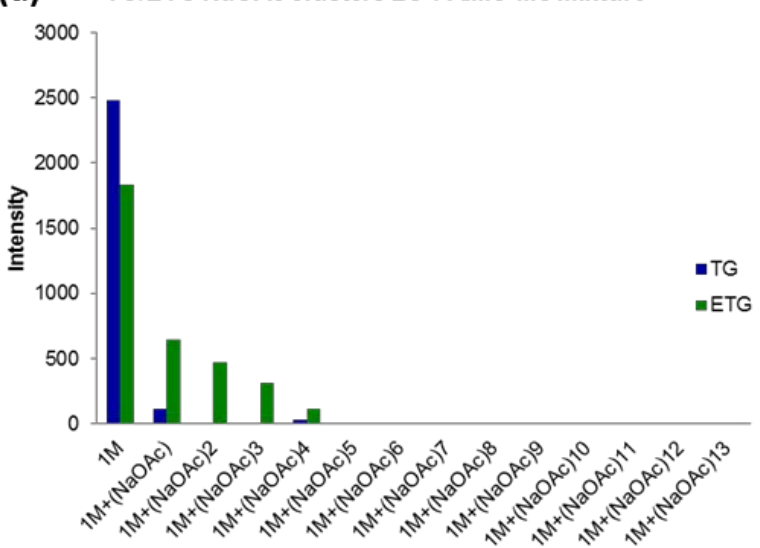

Supplementary Figure 2: Cluster patterns observed in the mass spectra for the doubly sodiated $[\mathrm{M}+2 \mathrm{Na}-\mathrm{H}]^{+}$ions of TG/ETG $(\mathrm{m} / \mathrm{z}$ 509.212) analyzed individually using (a) MS, (b) FAIMS-MS (DF 260 Td, CF 1.35 Td (TG) and CF 2.05 Td (ETG)) and (c) LC-FAIMS-MS; (d) LC-FAIMS-MS for TG/ETG analyzed as a mixture 
(a)

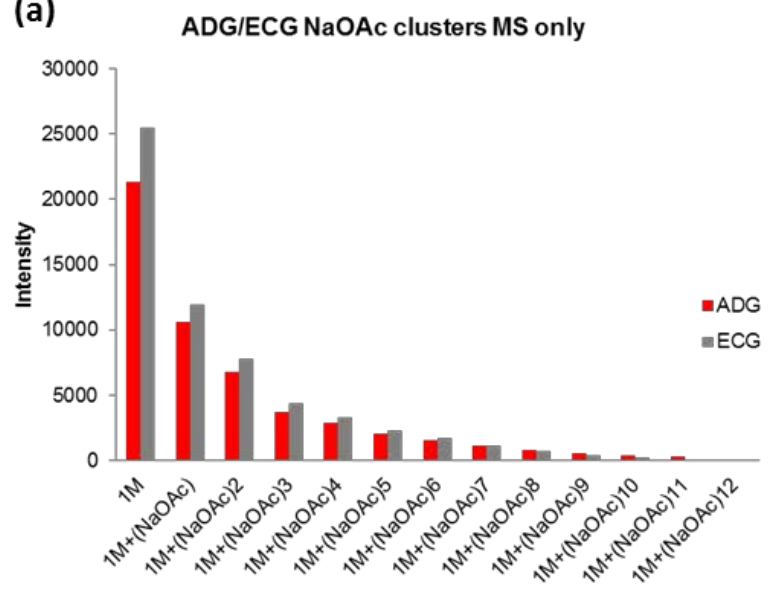

(c) ADG/ECG NaOAc clusters LC-FAIMS-MS individual

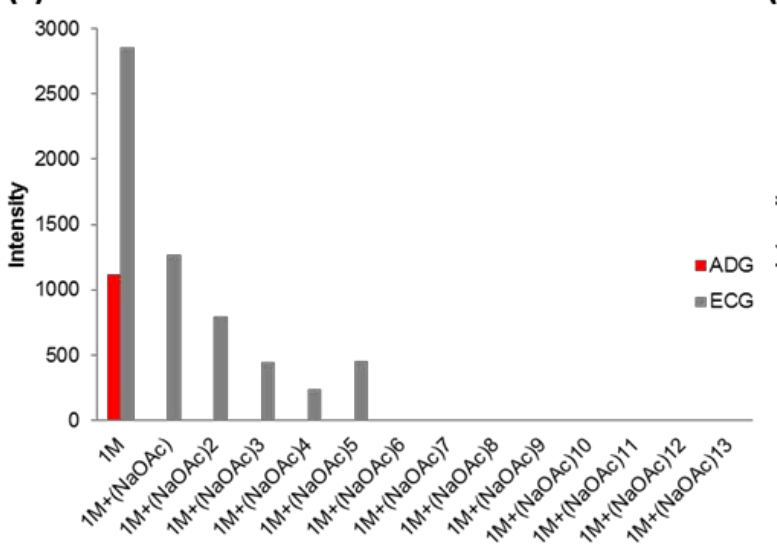

(b) ADG/ECG NaOAc clusters FAIMS-MS

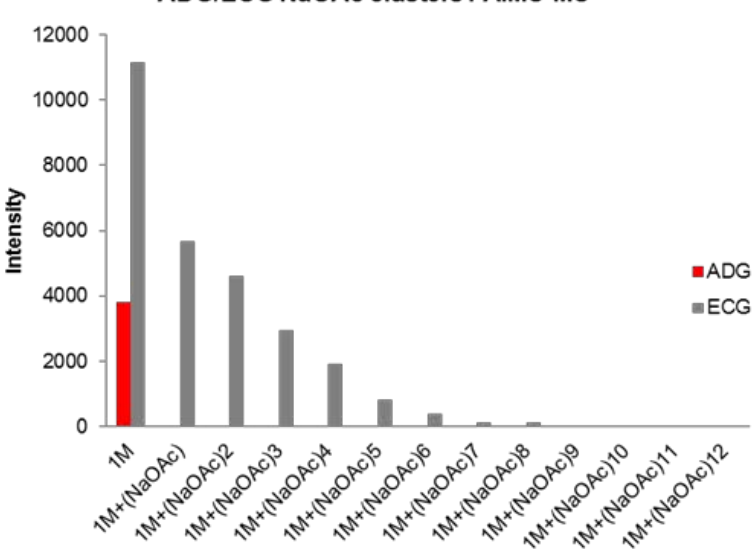

(d) ADG/ECG NaOAc clusters LC-FAIMS-MS mixture

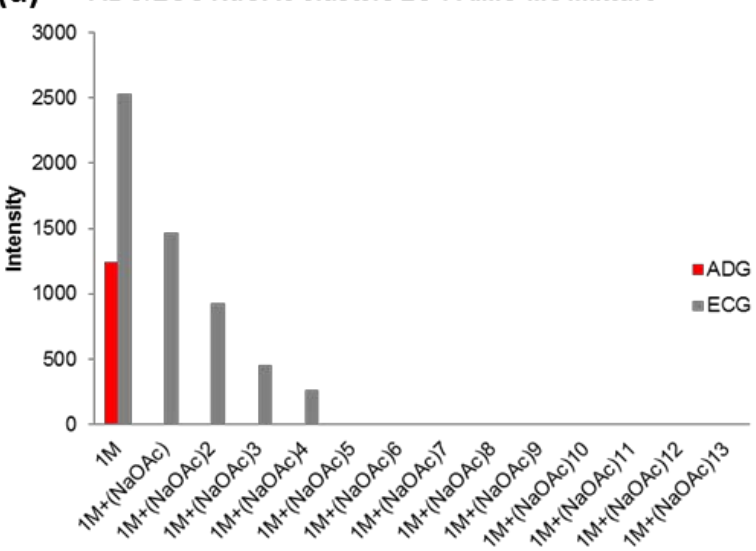

Supplementary Figure 3: Cluster patterns observed in the mass spectra for the doubly sodiated $[\mathrm{M}+2 \mathrm{Na}-\mathrm{H}]^{+}$ions of ADG/ECG ( $\mathrm{m} / \mathrm{z}$ 511.228) analyzed individually using (a) MS, (b) FAIMS-MS (DF 260 Td, CF 2.70 Td (ADG) and CF 2.05 Td (ECG)) and (c) LC-FAIMSMS; (d) LC-FAIMS-MS for ADG/ECG analyzed as a mixture 